\title{
Correlation between Bulk Thermodynamic Measurements and the Low-Temperature-Resistance Plateau in $\mathrm{SmB}_{6}$
}

\author{
W. A. Phelan, ${ }^{1,2}$ S. M. Koohpayeh, ${ }^{2}$ P. Cottingham, ${ }^{1,2}$ J. W. Freeland, ${ }^{3}$ J. C. Leiner, ${ }^{4}$ C. L. Broholm, ${ }^{2}$ and T. M. McQueen ${ }^{1,2^{*}}$ \\ ${ }^{1}$ Department of Chemistry, Johns Hopkins University, Baltimore, Maryland 21218, USA \\ ${ }^{2}$ Institute for Quantum Matter, Department of Physics and Astronomy, Johns Hopkins University, \\ Baltimore, Maryland 21218, USA \\ ${ }^{3}$ Advanced Photon Source, Argonne National Laboratory, Argonne, Illinois 60439, USA \\ ${ }^{4}$ Quantum Condensed Matter Division, Oak Ridge National Laboratory, \\ Oak Ridge, Tennessee 37831, USA
}

(Received 10 March 2014; revised manuscript received 10 June 2014; published 22 July 2014)

\begin{abstract}
Topological insulators are materials characterized by dissipationless, spin-polarized surface states resulting from nontrivial band topologies. Recent theoretical models and experiments suggest that $\mathrm{SmB}_{6}$ is the first topological Kondo insulator, in which the topologically nontrivial band structure results from electron-electron interactions via Kondo hybridization. Here, we report that the surface conductivity of $\mathrm{SmB}_{6}$ increases systematically with bulk carbon content. Further, addition of carbon is linked to an increase in $n$-type carriers, larger low-temperature electronic contributions to the specific heat with a characteristic temperature scale of $T^{*}=17 \mathrm{~K}$, and a broadening of the crossover to the insulating state. Additionally, $\mathrm{X}$-ray absorption spectroscopy shows a change in Sm valence at the surface. Our results highlight the importance of phonon dynamics in producing a Kondo insulating state and demonstrate a correlation between the bulk thermodynamic state and the low-temperature resistance of $\mathrm{SmB}_{6}$.
\end{abstract}

DOI: 10.1103/PhysRevX.4.031012

\section{INTRODUCTION}

Topological insulators are materials in which a nontrivial topology of the bulk band structure gives rise to spinmomentum locked surface states at interfaces with normal insulators [1,2]. In addition to being spin polarized, the surface states are protected from scattering by nonmagnetic disorder. These remarkable characteristics make topological insulators ideal building blocks for breakthroughs in many fields, including spintronics and quantum computation [3]. The theory and experimental consequences of a nontrivial band topology in weakly correlated, inversion symmetric materials such as $\mathrm{Sb}_{2} \mathrm{Te}_{3}, \mathrm{Bi}_{2} \mathrm{Te}_{3}$, and $\mathrm{Bi}_{2} \mathrm{Se}_{3}$ are now well established [4-6].

While topologically protected surface states in strongly correlated materials are an exciting possibility with theoretical backing, the experimental situation is less clear. $\mathrm{SmB}_{6}$ is a long-studied Kondo insulator $[7,8]$ that has come back into focus following the theoretical predictions that it may harbor topologically protected surface states below its Kondo temperature $\left(T_{K}\right)$ due to the interplay of spin-orbit

\footnotetext{
*mcqueen@jhu.edu
}

Published by the American Physical Society under the terms of the Creative Commons Attribution 3.0 License. Further distribution of this work must maintain attribution to the author(s) and the published article's title, journal citation, and DOI.
Subject Areas: Condensed Matter Physics,

Strongly Correlated Materials,

Topological Insulators coupling with strong electron-electron interactions [9-11]. Most samples of $\mathrm{SmB}_{6}$ show a resistance plateau at low temperature $(\sim 5-10 \mathrm{~K})$. Previous literature hypothesized that this low-temperature remnant metallicity originates from in-gap impurity states due to imperfect sample stoichiometry and secondary phases [12,13]. More recently, well-designed electrical transport measurements performed using flux-grown single crystals of $\mathrm{SmB}_{6}$ have shown that the low-temperature-resistivity plateau is due to surface, not bulk, conduction, and may be topological in origin [14-17]. An extensive range of additional measurements, including normal and spin-resolved angle resolved photoemission spectroscopy [18-24], surface circular dichroism [20], STM [25,26], and torque magnetometry [27], provide additional evidence for surface states.

Here, we report the results of both surface and bulk physical properties measurements on single crystals of $\mathrm{SmB}_{6}$ prepared using the floating zone (FZ) technique, both undoped and containing small levels of aluminum and carbon. We find that greater residual conduction at low temperature (i.e., more plateaued resistance) correlates with increased carbon content. Further, we find that the level of carbon systematically affects the Seebeck coefficient and specific heat. In addition to resolving a number of discrepancies in previous $\mathrm{SmB}_{6}$ studies, these results establish a correlation between the bulk thermodynamic state and the surface conduction of $\mathrm{SmB}_{6}$. Finally, we 
provide evidence through $\mathrm{x}$-ray absorption spectroscopy (XAS) and $\mathrm{x}$-ray magnetic circular dichroism (XMCD) that the surface of FZ-grown $\mathrm{SmB}_{6}$ is paramagnetic and that the valence state of Sm differs between the surface and the bulk.

\section{METHODS}

Single crystals of $\mathrm{SmB}_{6}$ with approximate dimensions of 30-mm length and 6-mm diameter are grown from rods of polycrystalline $\mathrm{SmB}_{6}$ (Testbourne Ltd., 99.9\%) using a four-mirror optical FZ furnace (Crystal Systems Inc., FZ-T$12000-X-V P O-P C$ ) with $4 \times 3 \mathrm{~kW}$ Xe lamps. For undoped $\mathrm{SmB}_{6}$, separate crystals are prepared with $\mathrm{SmB}_{6}$ from Testbourne Ltd. (99.5\%) and Alfa Aesar (AA 99.9\%) and found to exhibit similar low-temperature resistances. In order to simulate an "aluminum flux" growth of $\mathrm{SmB}_{6}$, a pressed pellet with a targeted mass of $\sim 0.20 \mathrm{~g}$ is prepared with a $\mathrm{SmB}_{6}$ (AA 99.9\%):Al (AA 99.97\%) mixture in a ratio of 50:50 wt $\% 50$. The prefabricated $\mathrm{SmB}_{6} / \mathrm{Al}$ pellet is placed between the seed and the feed rods and passed through the molten zone during the growth procedure. For the carbon-incorporated growth, the same procedure is followed with a pressed pellet prepared from a $0.5 \mathrm{~g}$ prehomogenized $\mathrm{SmB}_{6}$ (AA 99.9\%):C(AA 99.9995\%) mixture in a ratio of 15:85 wt $\%$. This pellet is arc melted, turned over, and remelted in a Zr-gettered argon atmosphere to ensure homogeneity. The mass loss of the resultant boule was determined to be negligible. For all growths, the molten zone is passed upwards with the feed rod being at the top and the seed rod below it and the pellets. One pass was sufficient to incorporate aluminum and carbon throughout each grown crystal. The mirrors are held in fixed positions and both rods are translated downwards. Growths are carried out under flowing ultrahigh purity Ar at a pressure of 2 bar and a flow rate of $2 \mathrm{~L} / \mathrm{min}$. The rotation rates for the seed (growing crystal) and the feed rod are 10 and $3 \mathrm{rpm}$, respectively. The overall growth rate for both experiments is $10 \mathrm{~mm} / \mathrm{h}$. Only one zone pass was performed for each growth. Slices of the crystals, close to the [100] orientation, are cut using a diamond saw.

Laboratory powder $\mathrm{x}$-ray diffraction is performed using $\mathrm{Cu} K \alpha$ radiation on a Bruker D8 Focus diffractometer with a LynxEye detector. A Si standard, with lattice parameter $a=5.43102 \AA$, is used in all samples to obtain accurate relative unit cell parameters. Rietveld refinements are performed in TOPAS (Bruker AXS) to extract lattice parameters of $a=4.1342(1) \AA$ and $a=4.1338(1) \AA$ near the beginning and end of the growth for $\mathrm{SmB}_{6}: \mathrm{C}$, respectively.

To prepare a single crystal of $\mathrm{LaB}_{6}, \mathrm{La}$ (AA 99.9\%) and B (AA 99\%) are combined and pressed into $0.73 \mathrm{~g}$ pellets with a $15 \%$ mass excess of $B$. These pellets are individually arc melted using the same procedure described for the $\mathrm{SmB}_{6} / \mathrm{C}$ pellet and then combined and ground using a stainless-steel mortar and pestle. The resulting powder is pressed into a rod using a hydrostatic press. This rod is divided into two pieces, and the resulting rods are heated in a high-temperature vacuum furnace at $5^{\circ} \mathrm{C} / \mathrm{hr}$ to $1600^{\circ} \mathrm{C}$ and held for $8 \mathrm{~h}$. The sintered rods are used to grow a single crystal of $\mathrm{LaB}_{6}$ using the same experiential growth parameters described above for the $\mathrm{SmB}_{6}$ growths.

An Amray 1810 SEM is used to collect images of the $\mathrm{SmB}_{6} / \mathrm{C}$ samples. An accelerating voltage of $30 \mathrm{kV}$ and a beam current of $40 \mu \mathrm{A}$ are used to collect images of naturally cleaved sample surfaces. Images are collected using both secondary and backscattering modes.

Resistance and Seebeck data are obtained using the resistivity and thermal transport options of a 9-T Quantum Design Physical Property Measurement System (PPMS). Unless otherwise stated, all measurements are performed using standard four-probe techniques, with approximate sample dimensions of length $\sim 1 \mathrm{~mm}$ and cross-sectional area $\sim 3 \mathrm{~mm}$ (thickness $\sim 0.8 \mathrm{~mm}$ ). Excitation currents for the resistance measurements are set sufficiently low to avoid significant Joule heating (see Fig. S5 in the Supplemental Material [28]). Pt leads are used for the resistance measurements, and Au-plated $\mathrm{Cu}$ leads are used for Seebeck measurements. The leads are mounted onto the samples using silver epoxy. All reported specific heat data are collected using the semiadiabatic pulse technique as implemented for the PPMS. Finally, all reported magnetization data were collected using the ACMS option of the PPMS.

The XAS and XMCD experiments are conducted at beam line 4-ID-C of the Advanced Photon Source located at Argonne National Laboratory. Surface-sensitive spectra are collected using total electron yield and bulk sensitive data with partial fluorescence yield using circularly polarized $x$ rays with a grazing $10^{\circ}$ configuration. The XMCD spectra are given by the difference between the absorption spectra of the right and left circularly polarized $\mathrm{x}$ rays. Measurements are performed at $T=10 \mathrm{~K}$ with an applied magnetic field of $\mu_{0} \mathrm{H}=5 \mathrm{~T}$ in the plane of the sample.

\section{RESULTS AND DISCUSSION}

Figure 1(a) shows the temperature-dependent resistance data from a high-resistance pure $\mathrm{SmB}_{6}$ sample, with a thickness of $1000 \mu \mathrm{m}$. This sample has a resistance that continues to increase down to at least $T=2.7 \mathrm{~K}(R>40 \Omega$ at $T=2.7 \mathrm{~K}, R / R_{300 \mathrm{~K}}>50000, R_{\square}>100 \Omega$ ). No plateau is observed below $\sim 5 \mathrm{~K}$ for this sample, unlike flux-grown samples of $\mathrm{SmB}_{6}[8,12,14-16]$. To test for a thickness dependence of the resistance behavior, as has previously been observed, Fig. 1(b) shows the resistance data of $\mathrm{SmB}_{6}$ from a different growth, measured with two different thicknesses, $800 \mu \mathrm{m}$ (nonthinned) and $48 \mu \mathrm{m}$ (thinned). Again, the resistance continues to diverge to the lowest temperatures measured $(T=2 \mathrm{~K})$, although 

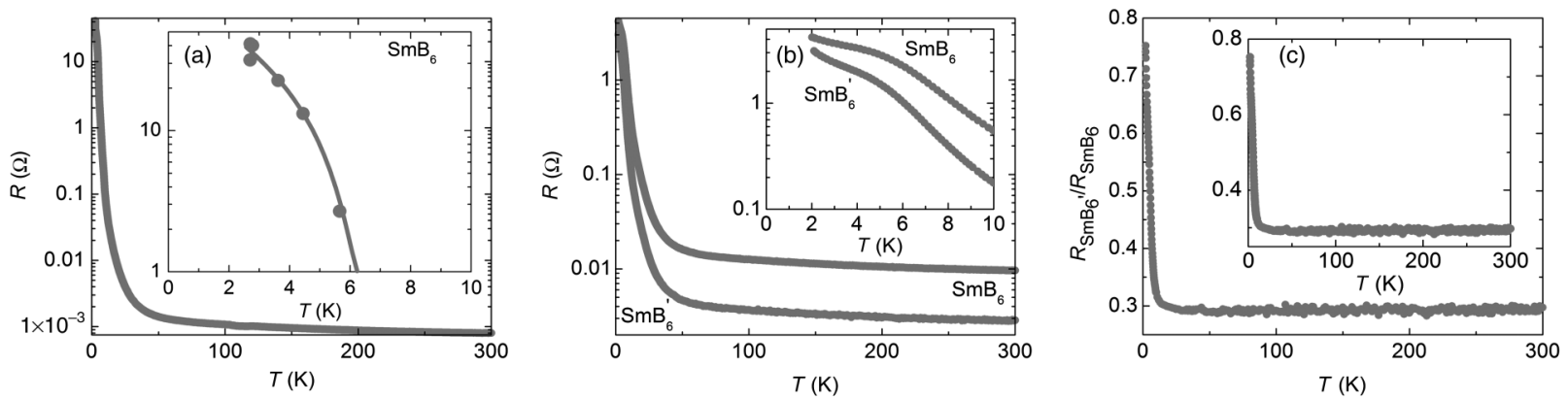

FIG. 1. (a) $R(T)$ data for the highest resistance $\mathrm{SmB}_{6}$ samples, thickness $1.01 \mathrm{~mm}$. It was not possible to measure below $T=2.7 \mathrm{~K}$

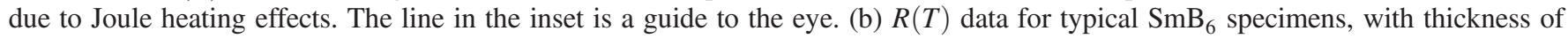
$\sim 800 \mu \mathrm{m}\left(\mathrm{SmB}_{6}{ }^{\prime}\right)$ and $\sim 48 \mu \mathrm{m}\left(\mathrm{SmB}_{6}\right)$. The inset shows that the resistances continue to diverge to the lowest temperatures measured $(2 \mathrm{~K})$, although there is a clear "knee" at $\sim 5 \mathrm{~K}$. (c) Resistance of a nonthinned sample of $\mathrm{SmB}_{6}\left(R_{\mathrm{SmB}_{6}}\right.$ ') normalized by the resistance of a thinned sample of $\mathrm{SmB}_{6}\left(R_{\mathrm{SmB}_{6}}\right)$ versus temperature. There is a deviation from a constant value at low temperatures, suggesting a switch away from three-dimensional dominated conduction below $\sim 5 \mathrm{~K}$.

there is a clear "knee" at $\sim 5 \mathrm{~K}$. If the conduction for the nonthinned and thinned samples is three dimensional, then $\rho_{\text {nonthinnned }}=\rho_{\text {thinned }}$ or $R_{\text {nonthinned }} \frac{\left(A_{\text {nonhthined }}\right)}{L_{\text {nonthimed }}}=$ $R_{\text {thinned }} \frac{\left(A_{\text {thinned }}\right)}{L_{\text {thinned }}}$. Therefore, $\frac{R_{\text {nonthinned }}}{R_{\text {thinned }}}$ should equal a constant factor. Figure 1(c) shows this ratio of the resistances. It deviates from a constant value at a temperature close to the knee. This is consistent with previous reports that have interpreted the change in slope as a switch from bulk to surface-dominated conduction [14,15]. Unlike previous reports, however, we do not observe true plateauing of the low-temperature resistance. Instead, a two-channel fit suggests a small semiconducting gap of 16(4) $\mathrm{K}$ for the low-temperature channel (Table SI, see Supplemental Material [28]).
To test if aluminum, the flux typically used to grow $\mathrm{SmB}_{6}$, is the origin of a more significant plateau, a set of samples from a simulated "aluminum flux" growth are prepared. Normalized resistance data for these samples compared to pure $\mathrm{SmB}_{6}$ are shown in Fig. 2(a). Samples grown in the presence of aluminum show a substantial reduction in the normalized resistance. Further, there is a gradual plateauing below $T=5 \mathrm{~K}$. However, there is no systematic trend with respect to position of the slices cut from the $\mathrm{SmB}_{6} / \mathrm{Al}$ crystal. These observations are consistent with filamentary inclusions of aluminum that provide a low-resistance pathway and "short out" the insulating bulk at low temperature.

However, incorporation of aluminum does not produce the dramatic resistance plateaus well known in this
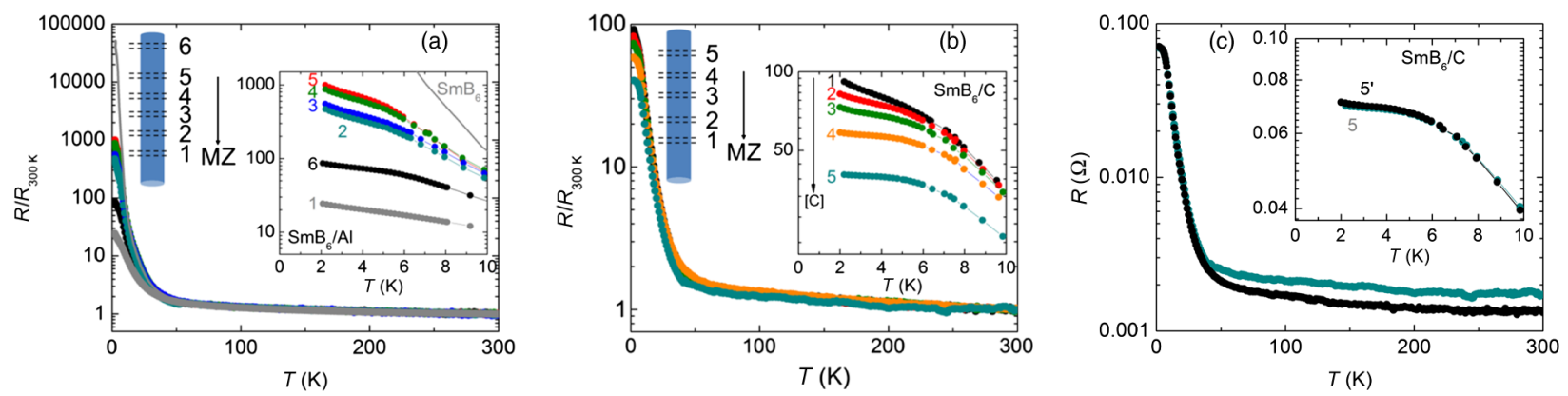

FIG. 2. Normalized resistance measurements for (a) $\mathrm{SmB}_{6} / \mathrm{Al}$ and (b) $\mathrm{SmB}_{6} / \mathrm{C}$. Both insets highlight the $R / R_{300 \mathrm{~K}}$ data below $T=10 \mathrm{~K}$. The colored numbers in the insets are arbitrary designations representing the position of the crystal slice along the $\mathrm{SmB} / \mathrm{Al}$ and $\mathrm{SmB}_{6} / \mathrm{C}$ crystals (MZ, molten zone). All samples are of comparable geometry (see Sec. II, $\pm 10 \%$ each dimension) to minimize sample shape effects that might arise due to a mixture of surface and bulk conductivity. The slope of the low-temperature resistance changes systematically with the position of the slice cut along the $\mathrm{SmB}_{6} / \mathrm{C}$ crystal, but not with aluminum position, indicating that variation of the bulk carbon content can be used to systematically tune the low-temperature-resistance plateau. The gray line shows the normalized resistance of undoped $\mathrm{FZ} \mathrm{SmB}_{6}$ in (a) and is off the scale of the inset in (b). (c) A comparison of the high- and low-temperature resistance of thinned (5) and a nonthinned (5') sample $\mathrm{SmB}_{6} / \mathrm{C}-5$. The resistance values at 2 and $300 \mathrm{~K}$ for the two samples change by $1.9 \%$ and $26 \%$, respectively. This demonstrates that the low-temperature plateau is independent of sample thickness, which is consistent with surface conduction. The sample is thinned without removal of the measurement leads, so all factors except thickness are held constant. 
system. The difference between our FZ-grown crystals and those previously reported provides a possible explanation. Previous $\mathrm{SmB}_{6}$ single crystals prepared using the $\mathrm{FZ}$ procedure have been reported to exhibit a low-temperature-resistance plateau $[13,29,30]$. The most recent work that describes the crystal growth in detail states that the carbon-based binders polyvinyl alcohol and polyvinyl butyral were used to prepare the rods necessary for the FZ growth [31], whereas no binder was used during our preparations. Further, carbon is also a common impurity in both boron and aluminum, and is difficult to quantify analytically because most methods have carbon present elsewhere (e.g., to affix the sample to the stage for energy or wavelength dispersive $\mathrm{x}$-ray spectroscopy).

To test whether the presence of carbon might be influential in producing a low-temperature-resistance plateau, we prepare $\mathrm{SmB}_{6}$ crystals containing carbon. The temperature-dependent normalized resistance data for these samples are shown in Fig. 2(b). The most remarkable observation is the systematic appearance of a resistance plateau below $T=6 \mathrm{~K}$, which becomes less prominent as a function of passed molten zone position. This observation suggests that the carbon content is varied systematically throughout the $\mathrm{SmB}_{6} / \mathrm{C}$ crystal. To test whether this plateau has the same origin in surface conductivity as previously reported [32], a measurement is performed on sample 5 before it is thinned (5'). Another resistance measurement is performed using sample 5 by polishing 5' without removing the electrical contacts, Fig. 2(c). The resistance values at 2 and $300 \mathrm{~K}$ for the two samples change by $1.9 \%$ and $26 \%$, respectively. This demonstrates that the low-temperature plateau is independent of sample thickness, which is consistent with surface-dominated conduction at low temperature. These results show that this well-known yet not well-understood feature can be controlled by chemical modification, specifically by adding carbon.

Suspecting that microstructural differences might be responsible for the dependence of surface conduction in carbon-containing $\mathrm{SmB}_{6}$, SEM micrographs (not shown) on naturally exposed surfaces of samples 1-5 are collected. No observable changes in the microstructure between samples are observed down to $\sim 50 \mathrm{~nm}$.

To further elucidate the origin of the dependence of the normalized resistance plateau on carbon content, we perform Seebeck, magnetization and specific heat measurements. The results of our Seebeck measurements are similar to those previously reported for $\mathrm{SmB}_{6}$, both $\mathrm{FZ}$ and flux grown, where the sign of the Seebeck coefficient is negative (positive) below (above) $T=300 \mathrm{~K}[33,34]$. The temperature at which the Seebeck coefficient crosses zero corresponds to the point where thermally generated carriers cancel the effect of chemically doped carriers. As is shown in the inset of Fig. 3, there is a small but significant (Fig. S1, Supplemental Material [28]) shift of this

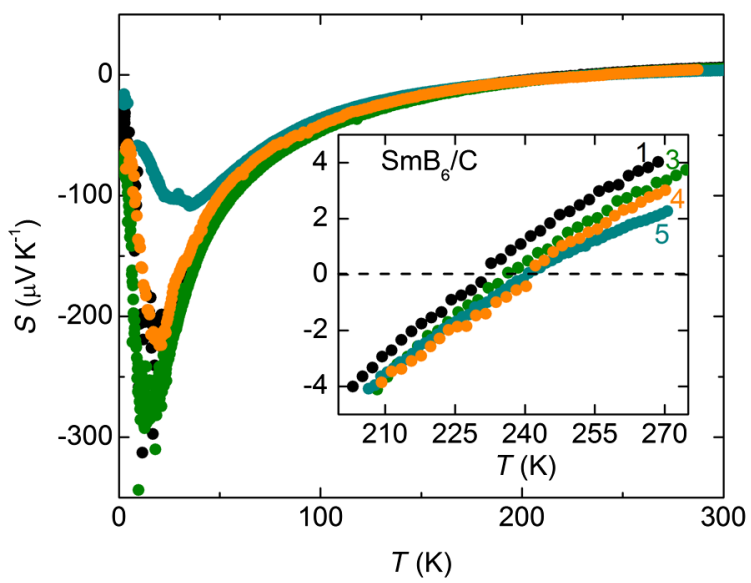

FIG. 3. Seebeck $(S)$ coefficient versus temperature for carboncontaining samples $1,3,4$, and $5 . T_{S=0}$, the temperature at which thermally excited carriers produce a change in the sign of $S$ from negative to positive upon heating, increases with position of the $\mathrm{SmB}_{6} / \mathrm{C}$ slice along the crystal.

cancellation to higher temperatures for different samples along the length of the $\mathrm{SmB}_{6} / \mathrm{C}$ crystal. This supports the conclusion that carbon results in the addition of bulk $n$-type carriers, expected if carbon replaces boron:

$$
\mathrm{B}_{\mathrm{B}}^{x}+\mathrm{C} \rightarrow \mathrm{C}_{\mathrm{B}}^{\cdot}+e^{-}+\mathrm{B} .
$$

This also suggests that the added carbon content is proportional to the magnitude of the numbers (i.e., the larger the number, the greater the carbon content).

Magnetization measurements also support a systematic change in electron count on addition of carbon. The magnetization data as a function of temperature is shown in Fig. 4(a). A Curie-Weiss analysis of the high-temperature $(100-300 \mathrm{~K})$ region yields an effective Curie constant that increases with sample number, Fig. 4(b) (Table SII, Supplemental Material [28]). This, along with the Seebeck data, suggests that the overall electron count is being altered upon carbon substitution.

Figure 5(a) shows the specific heat collected on pure and carbon-containing $\mathrm{SmB}_{6}$ specimens (2 and 5), as compared with isostructural, nonmagnetic $\mathrm{LaB}_{6}$. In all cases, a large excess heat capacity is observed. This corresponds to a large additional change in entropy in $\mathrm{SmB}_{6}$ that is not present in $\mathrm{LaB}_{6}$. The point of maximum entropy change occurs at $T \sim 40 \mathrm{~K}$, close to the temperature below which the resistance sharply increases. This change in entropy can be associated with the formation of the Kondo insulating state in $\mathrm{SmB}_{6}$. The corresponding Kondo temperature is then estimated to be $T_{K}=80-400 \mathrm{~K}$, depending on the approximation used to relate the peak in $C_{p} T^{-1}$ to $T_{K}$, in good agreement with previous literature [18-24]. By integrating the difference in $C_{p} T^{-1}$ between $\mathrm{SmB}_{6}$ and $\mathrm{LaB}_{6}$, it is possible to estimate the total entropy associated with this crossover [Fig. 5(b)]. We find that 

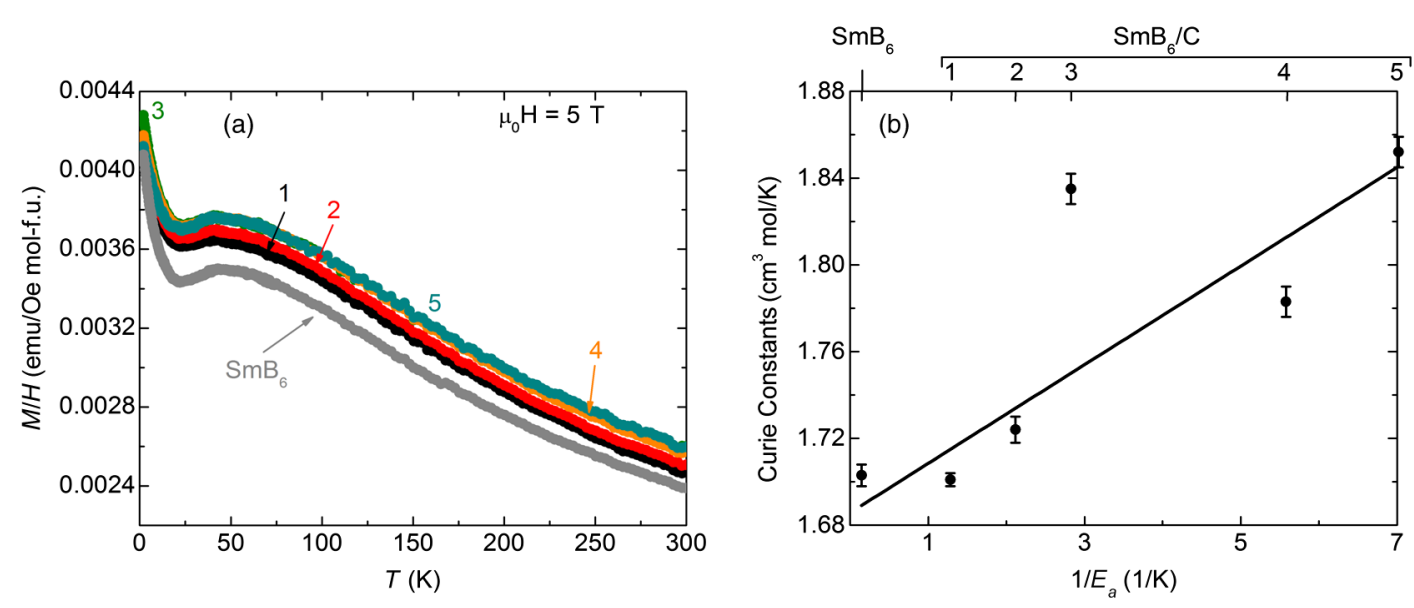

FIG. 4. (a) Magnetization data normalized by applied magnetic field $(M / H)$ as a function of temperature for $\mathrm{SmB}_{6}$ and $\mathrm{SmB}_{6} / \mathrm{C}-1-5$. (b) The Curie constant extracted from fits of the magnetization data from $T=100-300 \mathrm{~K} \mathrm{for} \mathrm{SmB}_{6} \mathrm{and} \mathrm{SmB}_{6} / \mathrm{C}-$ $1-5$ as a function of $1 / E_{a}$, a measure of the degree of plateauing of the low-temperature resistivity, defined to be $1 /$ slope $_{2-5} \mathrm{~K}$ of $\ln \left(R / R_{300 \mathrm{~K}}\right)$ versus $1 / T$. Tabulated values can be found in Table SII of the Supplemental Material [28].
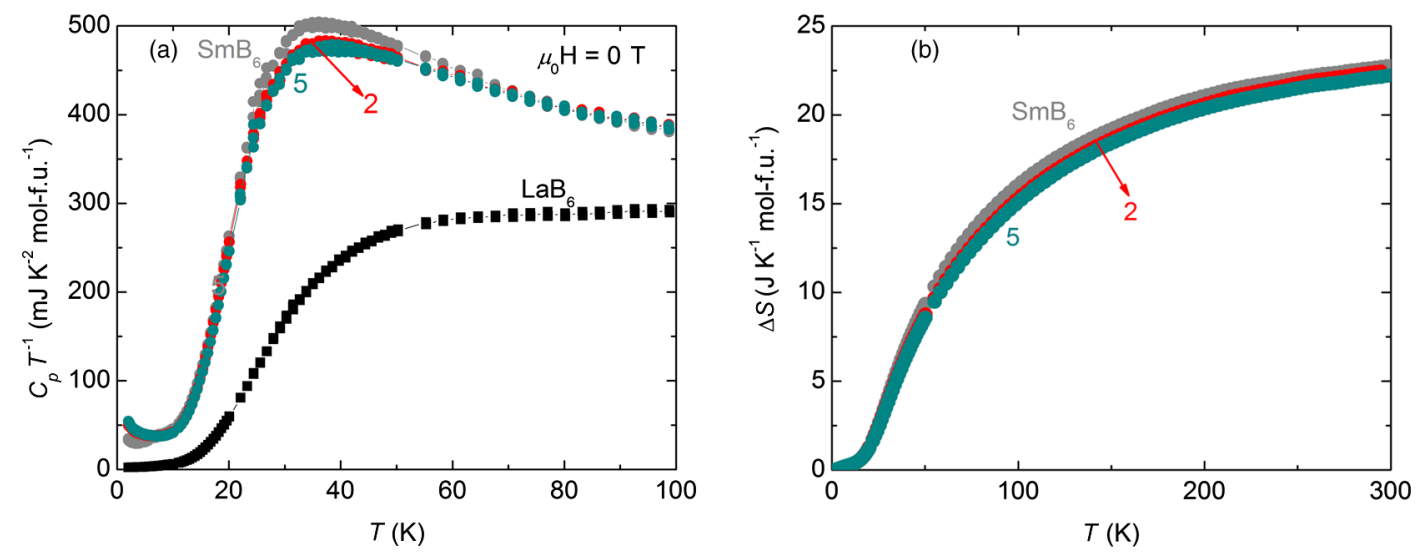

FIG. 5. (a) Specific heat divided by temperature $\left(C_{p} T^{-1}\right)$ versus temperature for pure $\mathrm{SmB}_{6}$, carbon-containing samples 2 and 5 , and the nonmagnetic analog $\mathrm{LaB}_{6}$. In addition to a low-temperature (around $T<10 \mathrm{~K}$ ) upturn that depends on carbon addition, there is a large excess specific heat that extends to room temperature. (b) Integration yields the changes in excess entropy; for all samples, the total is in excess of $2.3 R-2.9 R\left(R=8.314 \mathrm{JK}^{-1}\right.$ mol - f.u. $\left.{ }^{-1}\right)$.

the entropy change is $\Delta S \sim 23 \mathrm{JK}^{-1} \mathrm{~mol}-\mathrm{f} . \mathrm{u} .^{-1}=2.9 R$ $\left(R=8.314 \mathrm{JK}^{-1} \mathrm{~mol}-\mathrm{f} . \mathrm{u} .^{-1}\right)$, with the most stringent lower limit being $\Delta S \sim 19 \mathrm{JK}^{-1} \mathrm{~mol}-$ f.u. ${ }^{-1}=2.3 R$ depending the details of how the phonon background is subtracted using the nonmagnetic analog $\mathrm{LaB}_{6}$. This is substantially larger than the $\Delta S_{\mathrm{CF}} \sim 0.4 R$ expected based on the observed crystal field excitations in neutron scattering on powder samples that suggest only one thermally accessible set of crystal field levels at $\sim 28 \mathrm{meV}$ [35]. It is also greater than $\Delta S_{\mathrm{MV}} \sim 2.0 R$ expected for a $\mathrm{Sm}^{2+} / \mathrm{Sm}^{3+}$ mixed valence system in which the ratio is fixed at $1: 1$, as XAS and Sm-Mössbauer studies imply [36,37], and the accessible $J$ multiplets are $J=0,2$, and 3 for $\mathrm{Sm}^{2+}$ and $J=5 / 2$ for $\mathrm{Sm}^{3+}$, as previously suggested [7]. Some of the excess entropy will be due to the localization of the conduction electrons when the hybridization gap forms, but a more likely explanation for the large value of $\Delta S$ we observe is an additional phonon (lattice) contribution. This is similar to what has been observed by neutron scattering in $\mathrm{YbB}_{12}$ [38]. Further, a soft phonon mode is known to exist in $\mathrm{SmB}_{6}$ [39], and unusual changes in atomic displacement parameters and lattice parameters are known crystallographically $[40,41]$. This implies that understanding the phonon dynamics is essential to understanding $\mathrm{SmB}_{6}$. It also implies that understanding phonon dynamics will be critical in elucidating any topological properties of related hexaborides, e.g., $\mathrm{YbB}_{6}$, as has recently been suggested [42-44].

There are two noticeable changes in the specific heat data as a function of the $\mathrm{SmB}_{6} / \mathrm{C}$ sample. First, at temperatures in the range of the gap opening $(T \sim 40 \mathrm{~K})$, the peak broadens. This is consistent with a systematic addition of carbon defects found along the grown crystal, and these 

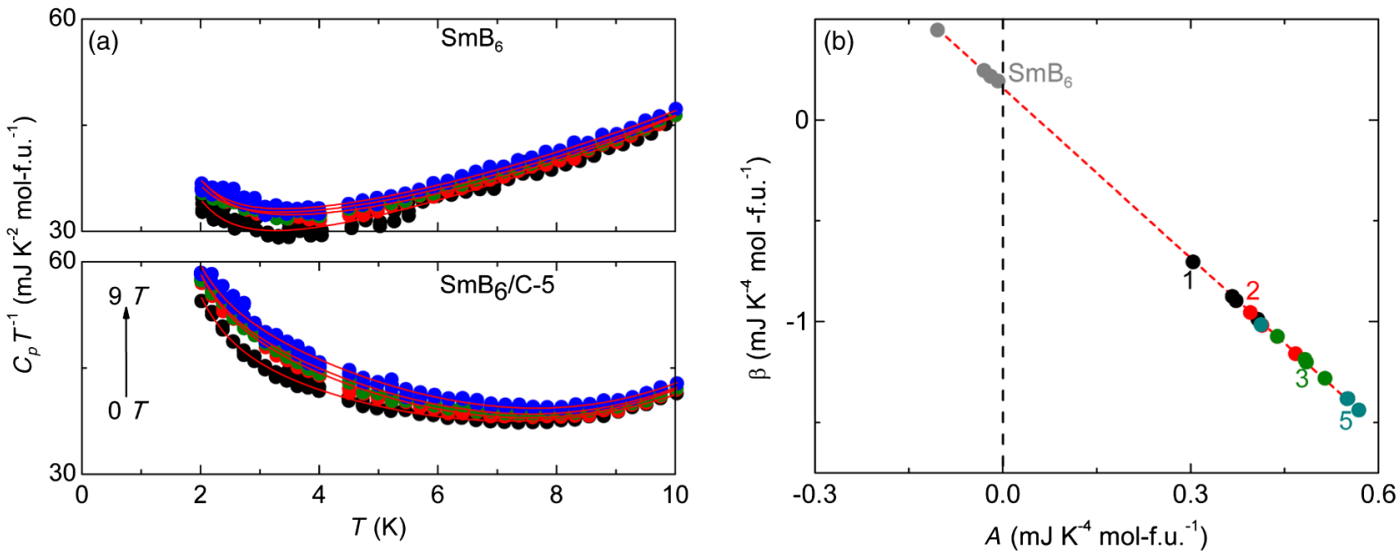

FIG. 6. (a) Specific heat divided by temperature for pure $\mathrm{SmB}_{6}$ and the carbon-containing sample 5 under applied fields of $\mu_{0} \mathrm{H}=0 \mathrm{~T}$ (black), $3 \mathrm{~T}$ (red), $5 \mathrm{~T}$ (green), and $9 \mathrm{~T}$ (blue). Model fits to the data at $T \leq 10 \mathrm{~K}$ (red line) are performed using the expression $C_{p} T^{-1}=\gamma+\beta T^{2}+A T^{2} \ln (T)+B T^{-3}$. (b) A plot of the $\beta$ versus $A$ coefficients shows that there is a universal linear relationship between the two parameters for all samples under all applied fields. The $y$ intercept corresponds to the lattice contribution to the specific heat, while the slope is given by $-\ln T^{*}$, where $T^{*}=17 \mathrm{~K}$ is a characteristic temperature for all samples and fields (see text).

defects have the effect of disrupting formation of the Kondo lattice. Second, there is an upturn in $C_{p} T^{-1}$ at $T<5 \mathrm{~K}$ that becomes more prominent, shown in more detail in Fig. 6(a). In each case, this upturn is sensitive to an applied magnetic field. We find that all specific heat data sets could be fit well to the expression

$$
C_{p}=\gamma T+\beta_{3} T^{3}+A T^{3} \ln \left(T / T^{*}\right)+B T^{-2} .
$$

The first and second terms are standard electronic and lattice contributions to the specific heat, respectively. The third term is typically associated with an exchangeenhanced paramagnetic metal [45], but is a correction to the $T$-linear electronic term that can occur in any Fermi liquid with momentum-dependent quasiparticle interactions [46], or electron-phonon coupling [47]. Such a term has been theoretically [48] and experimentally found to occur in heavy fermion systems [45]. Further, the heat capacity of $\mathrm{SmB}_{6}$ measured in a previous report shows this same $A T^{3} \ln \left(T / T^{*}\right)$ dependence down to $T \sim 0.1 \mathrm{~K}$ [13]. The fourth term, $B T^{-2}$, is the high-temperature expansion of a Schottky anomaly, and is only a minor contribution to specific heat, below $T \sim 2.8 \mathrm{~K}$ (Fig. S3, Supplemental Material [28]). To aid in the data-fitting procedure, Eq. (2) was recast in the following form:

$$
C_{p} T^{-1}=\gamma+\beta T^{2}+A T^{2} \ln (T)+B T^{-3},
$$

with $\beta=\beta_{3}-A \ln \left(T^{*}\right)$. The fits over the temperature range $T=2$ to $10 \mathrm{~K}$ are drawn in Fig. 6(a), and the resulting coefficients are tabulated in Table SIII of the Supplemental Material [28].

Remarkably, the $\beta$ and $A$ parameters from all samples (with and without carbon) lie on a universal curve, Fig. 6(b), with a single $\beta_{3}$ lattice contribution and a single characteristic temperature of $-\ln \left[T^{*}(\mathrm{~K})\right]=-2.8\left(T^{*}=\right.$ $17 \mathrm{~K})$ associated with Kondo hybridization. Test fits of the pure and $\mathrm{SmB}_{6} / \mathrm{C}-5$ samples over different temperature ranges $(T=3-12 \mathrm{~K}, 2-12 \mathrm{~K})$ show that, while there is some variability in the precise values of $\beta$ and $A$, depending on the range used, the values still lie on this universal curve. The $\beta_{3}$ lattice contribution extracted in this way corresponds to a Debye temperature of $\theta_{D}=230 \mathrm{~K}$ (we have followed the assumption typically made in the hexaboride literature, which assumes one atom per mole of material [i.e., per mol $(\mathrm{La} / \mathrm{Sm}) \mathrm{B}_{6}$ ] [49]). This is in good agreement with the previously reported $\theta_{D} \sim 250 \mathrm{~K}$ of $\mathrm{LaB}_{6}$, which corresponds to a local La mode [49].

Figure 7(a) shows key parameters from the Seebeck and specific heat measurements plotted versus $1 / E_{a}$, a measure of the degree of plateauing of the low-temperature resistivity, defined as $1 / E_{\mathrm{a}}=\lim _{T \rightarrow 0}\left[1 / T \ln \left(R / R_{300 \mathrm{~K}}\right)\right]$ (Fig. S4, Supplemental Material [28]). The negative-topositive Seebeck crossover, $T_{s=0}$ (left), the coefficient $\gamma$, and the temperature of the minimum value of the $C_{p} / T$ (right) increase concomitantly with $1 / E_{a}$. This demonstrates a correlation between the bulk electronic state and surface conductivity in $\mathrm{SmB}_{6}$ (surface contributions to the specific heat constitute $\ll 1 \%$ of the observed values).

Figure 7(b) diagrams a possible mechanism by which changes in the bulk can have such a significant impact on surface conduction. The DOS in a Kondo insulator for $T \ll T_{K}$ consists of two sharp peaks, from hybridization of localized rare earth $f$ states and conduction states, separated by a small gap. Our specific heat data can be used to estimate the Fermi level $\left(E_{F}\right)$ of each sample on such a plot in the following way. First, the magnitude of the $\gamma T$ term is proportional to the DOS at $E_{F}$, so samples with larger $\gamma$ are located in regions of higher DOS. Second, the magnitude of the thickness independence low-temperature resistance is 

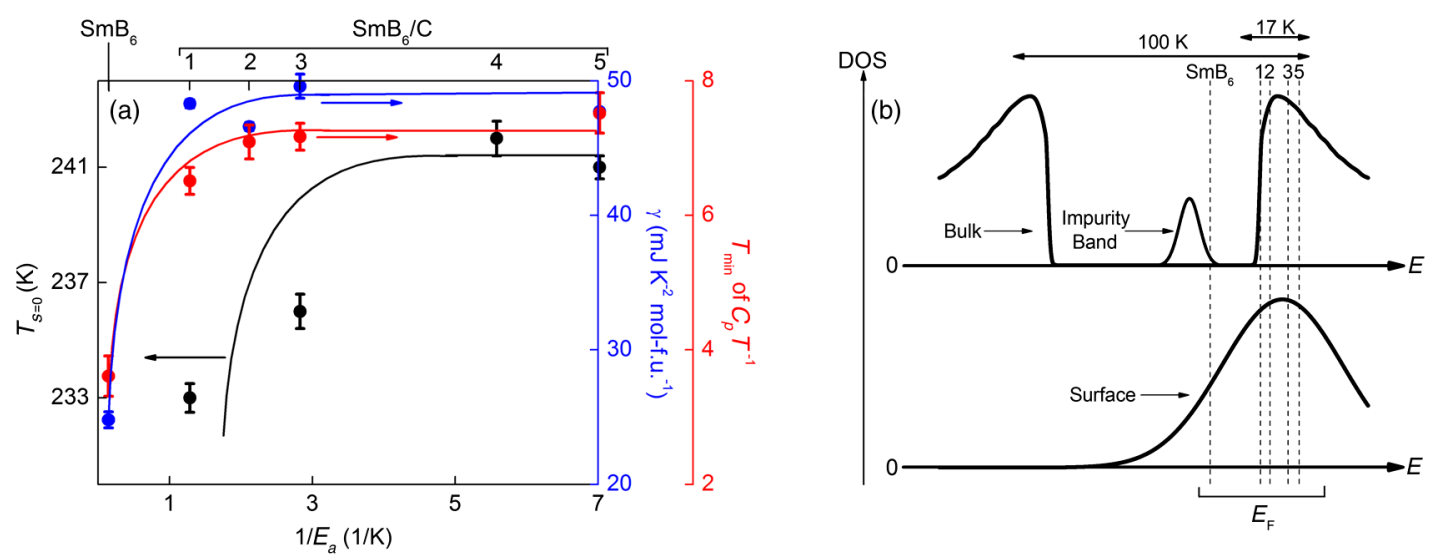

FIG. 7. (a) The negative-to-positive Seebeck crossover, $T_{s=0}$ (left), the Sommerfeld coefficient $\gamma$, and temperature of the minimum value of the $C_{p} / T$ (right) increase concomitantly with $1 / E_{a}$, a measure of the degree of plateauing of the low-temperature resistivity. Error bars represent the statistical portion of the error (see Table SIII, Supplemental Material [28]). (b) Schematic representation of the density of states of a Kondo insulator when $T \ll T_{K}$. The vertical lines show band-filling positions estimated from the specific heat (see text). Our finding of an increase in low-temperature conductivity (increased resistivity plateau) naturally follows if there are surface states near the hybridization gap.

higher when the band filling of surface states is lower. This places the Fermi level of undoped $\mathrm{SmB}_{6}$ in or close to the gap, while the carbon-containing samples have $E_{F}$ beyond the point along the band edge where the curvature changes from positive to negative. The $E_{F}$ positions estimated in this way are drawn as vertical dashed lines, and are consistent with the observed increase in $n$-type doping as carbon is added. If surface states are present in the neighborhood of the gap, the position of $E_{F}$ in the bulk acts to pin the band filling of the surface states, in turn modulating their conductivity. Critically, for undoped FZ $\mathrm{SmB}_{6}$, the bulk Fermi level is positioned such that the carrier density at the surface is low.

What, then, is the origin of this low-temperatureresistance plateau in $\mathrm{SmB}_{6}$ ? One possibility is that the plateau is a consequence of Joule heating of the sample. This explanation is unlikely, however, as resistance values obtained from zero-current extrapolation of IV curves agree with our low-excitation current measurements (see Figs. S5, S6, and S7 of the Supplemental Material [28]). Instead, in agreement with previous interpretations, a far more likely explanation is that it is due to surface conduction. Theoretical arguments suggest that surface states exist due to a nontrivial bulk band topology [9-11]. A second potential source of surface conduction is "normal" electronic surface states associated with the boron framework. A third possibility is a chemical change at the surface: it is well known that the surfaces of metal hexaborides can become metal deficient, especially in the case of $\mathrm{SmB}_{6}$, where surface vacancy patterns have been observed in low-energy electron diffraction and helium ion scattering spectroscopy [50]. This loss of Sm (and corresponding oxidation of some surface $\mathrm{Sm}^{2+}$ to $\mathrm{Sm}^{3+}$ ) naturally disrupts the Kondo lattice and coherence [51]. A fourth possibility is that surface reconstructions, such as those observed by STM [25,26], change the electronic structure at the surface. A fifth possibility suggested by our data, especially the ability to have the resistance plateau occur over a wide range of absolute resistance values and the previously reported dependence of the low-temperature plateau on cleaning and etching procedures, is that both topological and nontopological surface states are simultaneously present. The precise crystallographic orientations of surfaces where the normal and nontrivial surface states may coexist depends on the individual origins of each state. For example, if the normal surface states are associated with the boron framework and $\mathrm{SmB}_{6}$ belongs to the class of strong $Z_{2}$ topological insulators, then both normal and nontrivial states will coexist on all surfaces. However, if normal surface states are associated with the boron framework and $\mathrm{SmB}_{6}$ is a topological crystalline insulator, as has recently been predicted [52], then the coexistence of these states is limited to the (110) surface. Finally, if it is only polarity-driven surface reconstructions that give rise to normal surface states and $\mathrm{SmB}_{6}$ belongs to the class of strong $Z_{2}$ topological insulators, then the surface of coexistence is the (100). A sixth possibility is that $\mathrm{SmB}_{6}$ is poised at the boundary between normal and topological states and small changes in electron count push the system to one side or the other of the phase boundary. The estimated Sm valence of 2.5+, from XAS and Mössbauer measurements [36,37], is very close to the theoretical boundary of $2.56+$ [53], and this scenario would reconcile our results with theory and with previous experiments.

To understand the nature of the $\mathrm{SmB}_{6}$ surface in more detail, we collect $\mathrm{Sm}-M_{4,5}$ XAS and XMCD spectra simultaneously in surface (electron yield) and bulk (fluorescence yield) modes at $T=10 \mathrm{~K}$ and $\mu_{0} \mathrm{H}=5 \mathrm{~T}$ [Figs. 8(a) and 8(b)]. The bulk spectra are consistent with a mixture of $\mathrm{Sm}^{2+}$ and $\mathrm{Sm}^{3+}$, with no appreciable 

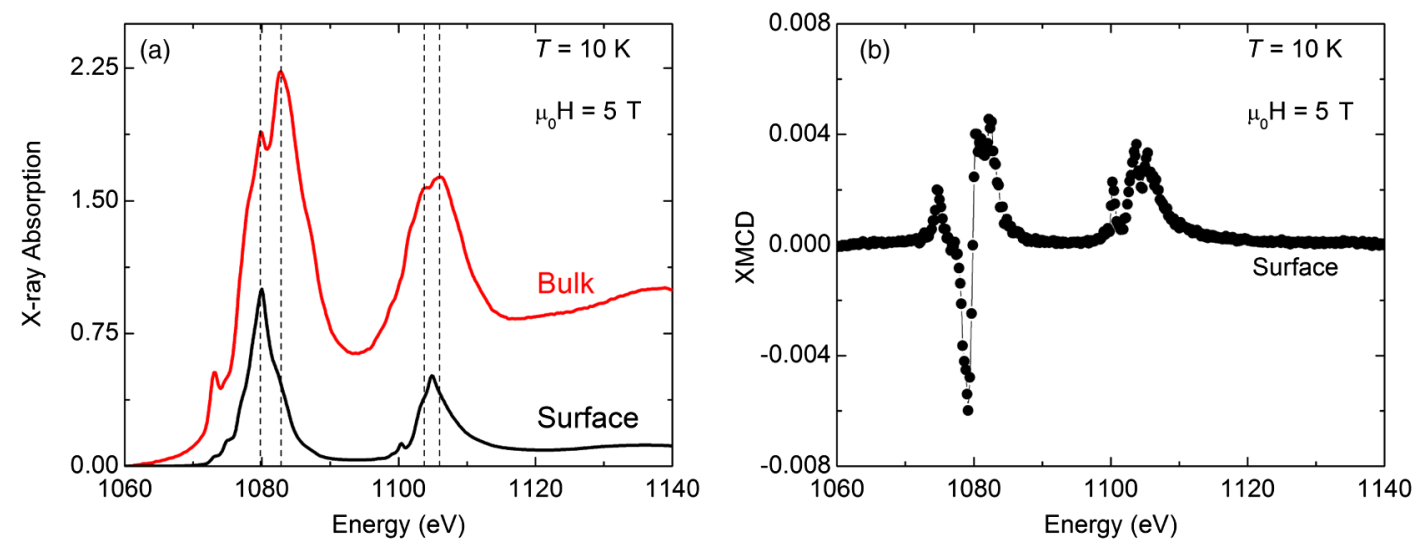

FIG. 8. (a) XAS and (b) XMCD spectra of surface and bulk $\mathrm{SmB}_{6}$ collected at the $\mathrm{Sm} M$ edge at $T=10 \mathrm{~K}$ and $\mu_{0} \mathrm{H}=5 \mathrm{~T}$. The bulk is consistent with previous reports of a mixed valence mixture of $\mathrm{Sm}^{2+}$ and $\mathrm{Sm}^{3+}$ oxidation states (dashed lines). In contrast, the surface of $\mathrm{SmB}_{6}$ contains entirely $\mathrm{Sm}^{3+}$ (dashed lines). The surface displays a nonzero XMCD signal consistent with the presence of paramagnetic $\mathrm{Sm}^{3+}$ ions.

magnetization, as previously reported [36,54]. In contrast, the surface consists of almost entirely $\mathrm{Sm}^{3+}$ and shows a discernible XMCD signal characteristic of a net magnetic moment. The magnitude of the surface XMCD response is small, approximately $1 / 10$ that observed in ferromagnetic $\mathrm{Sm}_{0.974} \mathrm{Gd}_{0.02} \mathrm{Al}_{2}$ [55]. Using sum rules [56,57], the estimated orbital $\left(M_{L}\right)$ and spin $\left(M_{S}\right)$ moments of $\mathrm{Sm}^{3+}$ at the surface are $0.09 \mu_{B}$ and $-0.05 \mu_{B}$, respectively. These values correspond to a net magnetic moment of $0.09 \mu_{B}$ at $T=$ $10 \mathrm{~K}$ and $\mu_{0} \mathrm{H}=5 \mathrm{~T}$, in good agreement with the $0.08 \mu_{B}$ expected for paramagnetic $\mathrm{Sm}^{3+}$ ions, implying the surface is paramagnetic at $T=10 \mathrm{~K}$ and distinct from the bulk Kondo insulator for which no induced magnetic moment was observed under these thermomagnetic conditions by XMCD.

The surface-versus-bulk comparison of the XAS data is definitive proof of changes in the electronic structure at the surface of $\mathrm{SmB}_{6}$. The disparate valences at the surface and bulk could give rise to a surface-to-bulk electric field and, hence, band bending at the interface, and would provide one possible explanation for the dependence of surface conduction on the bulk properties, but experiments such as liquid gating are needed to clarify this issue. Further, the nonzero surface XMCD indicates that magnetic ions are present on the surface. A particularly intriguing possibility that would explain the results of recent magnetotransport measurements [58] and the low-frequency conduction observed by terahertz spectroscopy [59] is that the surface of undoped $\mathrm{SmB}_{6}$ becomes magnetically ordered at even lower temperatures. Even if topologically protected surface states are present, such magnetic order would open a small gap in the surface density of states and remove the low-temperature surface conductivity. The action of carbon would then be to suppress the formation of magnetic order (e.g., by conversion of $J \neq 0 \mathrm{Sm}^{3+}$ to $J=0 \mathrm{Sm}^{2+}$ at the surface by pinning to the bulk Fermi level) and restore time-reversal symmetry. This scenario is consistent with two-channel fits of the resistance data, where the primary action of carbon is to reduce the effective activation gap of surface conduction, with no significant change in the magnitude of the bulk activation gap (Table SI, Supplemental Material [59]).

\section{CONCLUSIONS}

We find that there is a correlation between quantities obtained from bulk thermodynamic measurements and surface conduction in $\mathrm{SmB}_{6}$. Small aluminum inclusions do not dramatically affect the low-temperature surface conduction in FZ-grown samples. The $\Delta S \sim 23 \mathrm{JK}^{-1}$ mol - f.u. ${ }^{-1}$ entropy change through the Kondo coherence crossover at $T \sim 40 \mathrm{~K}$ is too large to originate solely from magnetic and electronic degrees of freedom, and implies that consideration of lattice vibrations and their coupling to magnetic and electronic states is critical to understanding $\mathrm{SmB}_{6}$. Further, the valence of $\mathrm{Sm}$ at the surface is found to be distinct from the bulk, indicative of a change in electronic structure at the surface. In short, our results demonstrate a correlation between bulk thermodynamic properties of $\mathrm{SmB}_{6}$ and the low-temperature-resistivity plateau arising from surface-dominated conduction, a necessary prerequisite for topological Kondo insulating behavior.

\section{ACKNOWLEDGMENTS}

We thank J. Checkelsky, N.P. Armitage, and O. Tchernyshyov for useful discussions, C. L. Chien for providing some sample materials, N. Laurita for sample manipulations, and N. Hartman for assistance with the SEM data collection. The work at IQM was supported by the U.S. Department of Energy, office of Basic Energy Sciences, Division of Material Sciences and Engineering under Grant No. DE-FG02-08ER46544. 
T. M. M. acknowledges support from the David and Lucile Packard Foundation. Work at Argonne National Laboratory and use of the Advanced Photon Source was supported by the U.S. Department of Energy, Office of Basic Energy Sciences under Contract No. DE-AC02-06CH11357.

[1] M. Z. Hasan and C. L. Kane, Colloquium: Topological Insulators, Rev. Mod. Phys. 82, 3045 (2010).

[2] H. C. Manoharan, Topological Insulators: A Romance with Many Dimensions, Nat. Nanotechnol. 5, 477 (2010).

[3] J. E. Moore, The Birth of Topological Insulators, Nature (London) 464, 194 (2010).

[4] H. J. Zhang, C. X. Liu, X. L. Qi, X. Dai, Z. Fang, and S. C. Zhang, Topological Insulators in $\mathrm{Bi}_{2} \mathrm{Se}_{3}, \mathrm{Bi}_{2} \mathrm{Te}_{3}$ and $\mathrm{Sb}_{2} \mathrm{Te}_{3}$ with a Single Dirac Cone on the Surface, Nat. Phys. 5, 438 (2009).

[5] D. Hsieh et al., Observation of Time-Reversal-Protected Single-Dirac-Cone Topological-Insulator States in $\mathrm{Bi}_{2} \mathrm{Te}_{3}$ and $\mathrm{Sb}_{2} \mathrm{Te}_{3}$, Phys. Rev. Lett. 103, 146401 (2009).

[6] Y. Xia et al., Observation of a Large-Gap TopologicalInsulator Class with a Single Dirac Cone on the Surface, Nat. Phys. 5, 398 (2009).

[7] J. C. Nickerson, R. M. White, K. N. Lee, R. Bachmann, T. H. Geballe, and G. W. Hull, Physical Properties of $\mathrm{SmB}_{6}$, Phys. Rev. B 3, 2030 (1971).

[8] J. C. Cooley, M. C. Aronson, Z. Fisk, and P. C. Canfield, $\mathrm{SmB}_{6}$ : Kondo Insulator or Exotic Metal?, Phys. Rev. Lett. 74, 1629 (1995).

[9] M. Dzero, K. Sun, V. Galitski, and P. Coleman, Topological Kondo Insulators, Phys. Rev. Lett. 104, 106408 (2010).

[10] T. Takimoto, $\mathrm{SmB}_{6}$ : A Promising Candidate for a Topological Insulator, J. Phys. Soc. Jpn. 80, 123710 (2011).

[11] M. Dzero, K. Sun, P. Coleman, and V. Galitski, Theory of Topological Kondo Insulators, Phys. Rev. B 85, 045130 (2012).

[12] A. Kebede et al., Studies of the correlated electron system $\mathrm{SmB}_{6}$, Physica (Amsterdam) 223-224, 256 (1996).

[13] S. Gabáni, K. Flachbart, E. Konovalova, M. Orendáč, Y. Paderno, V. Pavlík, and J. Šebek, Properties of the In-Gap States in $\mathrm{SmB}_{6}$, Solid State Commun. 117, 641 (2001).

[14] D. J. Kim, S. Thomas, T. Grant, J. Botimer, Z. Fisk, and J. Xia, Surface Hall Effect and Nonlocal Transport in $\mathrm{SmB}_{6}$ : Evidence for Surface Conduction, Sci. Rep. 3, 3150 (2013).

[15] D. J. Kim, J. Xia, and Z. Fisk, Topological Surface State in the Kondo Insulator Samarium Hexaboride, Nat. Mater. 13, 466 (2014).

[16] S. Thomas, D. J. Kim, S. B. Chung, T. Grant, Z. Fisk, and J. Xia, Weak Antilocalization and Linear Magnetoresistance in the Surface State of $\mathrm{SmB}_{6}$, arXiv:1307.4133.

[17] S. Wolgast, C. Kurdak, K. Sun, J. W. Allen, D.-J. Kim, and Z. Fisk, Low-Temperature Surface Conduction in the Kondo Insulator $\mathrm{SmB}_{6}$, Phys. Rev. B 88, 180405(R) (2013).

[18] S. Shigemas et al., Spin-Polarized Angle-Resolved Photoelectron Spectroscopy of the So-Predicted Kondo Topological Insulator $\mathrm{SmB}_{6}$, arXiv:1309.7839.

[19] M. Neupane et al., Surface Electronic Structure of a Topological Kondo Insulator Candidate $\mathrm{SmB}_{6}$ : Insights from High-Resolution ARPES, arXiv:1306.4634.
[20] J. Jiang et al., Observation of Possible Topological In-Gap Surface States in the Kondo Insulator $\mathrm{SmB}_{6}$ by Photoemission, Nat. Commun. 4, 3010 (2013).

[21] Z. H. Zhu, A. Nicolaou, G. Levy, N. P. Butch, P. Syers, X. F. Wang, J. Paglione, G. A. Sawatzky, I. S. Elfimov, and A. Damascelli, Polarity-Driven Surface Metallicity in $\mathrm{SmB}_{6}$, Phys. Rev. Lett. 111, 216402 (2013).

[22] J. D. Denlinger, J. W. Allen, J.-S. Kang, K. Sun, B.-L. Min, D.-J. Kim, and Z. Fisk, $\mathrm{SmB}_{6}$ Photoemission: Past and Present, arXiv:1312.6636.

[23] N. Xu et al., Surface and Bulk Electronic Structure of the Strongly Correlated System $\mathrm{SmB}_{6}$ and Implications for a Topological Kondo Insulator, Phys. Rev. B 88, 121102(R) (2013).

[24] E. Frantzeskakis et al., Kondo Hybridization and the Origin of Metallic States at the (001) Surface of $\mathrm{SmB}_{6}$, Phys. Rev. X 3, 041024 (2013).

[25] M. Yee, H. Yang, A. Soumyanarayanan, D. Kim, Z. Fisk, and J. Hoffman, Imaging the Kondo Insulating Gap on $\mathrm{SmB}_{6}$, arXiv:1308.1085.

[26] S. Rößler, T.-H. Jang, D.-J. Kim, L. H. Tjeng, Z. Fisk, F. Steglich, and S. Wirth, Hybridization Gap and Fano Resonance in $\mathrm{SmB}_{6}$, Proc. Natl. Acad. Sci. U.S.A. 111, 4798 (2014).

[27] G. Li et al., Ouantum Oscillations in Kondo Insulator $\mathrm{SmB}_{6}$, arXiv:1306.5221.

[28] See Supplemental Material at http://link.aps.org/ supplemental/10.1103/PhysRevX.4.031012 contains seven figures, three tables, a description of how the magnetic moment was extracted from the surface XMCD data, and supplemental references.

[29] M. Kasaya and F. Iga, Preparation and Physical Properties of Rare Earth Hexaborides and Dodecaborides, AIP Conf. Proc. 140, 11 (1986).

[30] K. Flachbart, S. Gabáni, E. Konovalova, Y. Paderno, and V. Pavlík, Ground State Formation in Intermediate Valent $\mathrm{SmB}_{6}$, Physica (Amsterdam) 293B, 417 (2001).

[31] M. C. Hatnean, M. R. Lees, D. M. Paul, and P. G. Balakrishana, Large, High Quality Single-Crystals of the New Topological Kondo Insulator, $\mathrm{SmB}_{6}$, Sci. Rep. 3, 3071 (2013).

[32] X. Zhang, N. P. Butch, P. Syers, S. Ziemak, R. L. Greene, and J. Paglione, Hybridization, Inter-Ion Correlation, and Surface States in the Kondo Insulator $\mathrm{SmB}_{6}$, Phys. Rev. X 3, 011011 (2013).

[33] N. E. Sluchanko, V. V. Glushkov, S. V. Demishev, A. A. Pronin, A. A. Volkov, M. V. Kondrin, A. K. Savchenko, and S. Kunii, Low-Temperature Transport Anisotropy and Many-Body Effects in $\mathrm{SmB}_{6}$, Phys. Rev. B 64, 153103 (2001).

[34] M. Takeda, T. Fukuda, F. Domingo, and T. Miura, Thermoelectric Properties of Some Metal Borides, J. Solid State Chem. 177, 471 (2004).

[35] P. A. Alekseev, V. N. Lazukov, R. Osborn, B. D. Rainford, I. P. Sadikov, E. S. Konovalova, and Y. B. Paderno, Neutron Scattering Study of the Intermediate-Valent Ground-State in $\mathrm{SmB}_{6}$, Europhys. Lett. 23, 347 (1993).

[36] J. M. Tarascon, Y. Isikawa, B. Chevalier, J. Etourneau, P. Hagenmuller, and M. Kasaya, Temperature Dependence of the Samarium Oxidation State in $\mathrm{SmB}_{6}$ and $\mathrm{Sm}_{1-x} \mathrm{La}_{x} \mathrm{~B}_{6}$, J. Phys. France 41, 1141 (1980). 
[37] R. L. Cohen, M. Eibschut, and K. W. West, Electronic and Magnetic Structure of $\mathrm{SmB}_{6}$, Phys. Rev. Lett. 24, 383 (1970).

[38] A. Bouvet, T. Kasuya, M. Bonnet, L. P. Regnault, J. RossatMignod, F. Iga, B. Fak, and A. Severing, Magnetic Excitations Observed by Means of Inelastic Neutron Scattering in Polycrystalline $\mathrm{YbB}_{12}$, J. Phys. Condens. Matter 10, 5667 (1998).

[39] P. A. Alekseev et al., Lattice Dynamics of Intermediate Valence Semiconductor $\mathrm{SmB}_{6}$ Europhys. Lett. 10, 457 (1989).

[40] V. A. Trounov, A. L. Malyshev, D. Y. Chernyshov, M. M. Korsukova, V. N. Gurin, L. A. Aslanov, and V. V. Chernyshev, Temperature Dependence of the Parameters of Atoms in the Crystal-Structure of the Intermediate-Valence Semiconductor $\mathrm{SmB}_{6}$ : Investigation by High-Resolution Powder Neutron-Diffraction, J. Phys. Condens. Matter 5, 2479 (1993).

[41] W. A. Phelan et al. (to be published).

[42] H. Weng, J. Zhao, Z. Wang, Z. Fang, and X. Dai, Topological Crystalline Kondo Insulator in Mixed Valence Ytterbium Borides, Phys. Rev. Lett. 112, 016403 (2014).

[43] M. Xia et al., Angle-Resolved Photoemission Spectroscopy Study on the Surface States of the Correlated Topological Insulator $\mathrm{YbB}_{6}$, arXiv:1404.6217.

[44] M. Neupane et al., Observation of Non-Kondo-like Electronic Structure in Strongly Correlated Electron System $\mathrm{YbB}_{6}$, arXiv:1404.6814.

[45] A. Tari, The Specific Heat of Matter at Low Temperatures (Imperial College Press, London, 2003), pp. 78-99.

[46] C. J. Pethick and G. M. Carneiro, Specific Heat of a Normal Fermi Liquid. I. Landau-Theory Approach, Phys. Rev. A 7, 304 (1973).

[47] A. V. Chubukov, D. L. Maslov, and A. J. Millis, Nonanalytic Corrections to the Specific Heat of a Three-Dimensional Fermi Liquid, Phys. Rev. B 73, 045128 (2006).

[48] J. Rasul and H. U. Desgranges, Specific Heat of the Anderson Lattice at Low Temperatures, J. Phys. C 19 , L671 (1986).
[49] D. Mandrus, B. C. Sales, and R. Jin, Localized Vibrational Mode Analysis of the Resistivity and Specific Heat of $\mathrm{LaB}_{6}$, Phys. Rev. B 64, 012302 (2001).

[50] M. Aono, R. Nishitani, C. Oshima, T. Tanaka, E. Bannai, and $\mathrm{S}$. Kawai, $\mathrm{LaB}_{6}$ and $\mathrm{SmB}_{6}$ (001) Surface Studied by Angle-Resolved XPS, LEED, and ISS, Surf. Sci. 86, 631 (1979).

[51] P. Schlottmann, Effects of Doping in Kondo Insulators, J. Appl. Phys. 75, 7044 (1994).

[52] M. Legner, A. Ruegg, and M. Sigrist, Topological Invariants, Surface States, and Interaction-Driven Phase Transitions in Correlated Kondo Insulators with Cubic Symmetry, Phys. Rev. B 89, 085110 (2014).

[53] V. Alexandrov, M. Dzero, and P. Coleman, Cubic Topological Kondo Insulators, Phys. Rev. Lett. 111, 226403 (2013).

[54] M. Mizumaki, S. Tsutsui, and F. Iga, Temperature Dependence of $\mathrm{Sm}$ Valence in $\mathrm{SmB}_{6}$ Studied by X-Ray Absorption Spectroscopy, J. Phys. Conf. Ser. 176, 012034 (2009).

[55] S. S. Dhesi, G. van der Laan, P. Bencok, N. B. Brookes, R. M. Galera, and P. Ohresser, Spin- and Orbital-Moment Compensation in the Zero-Moment Ferromagnet $\mathrm{Sm}_{0.974} \mathrm{Gd}_{0.026} \mathrm{Al}_{2}$, Phys. Rev. B 82, 180402 (2010).

[56] P. Carra, B. T. Thole, M. Altarelli, and X. Wang, X-Ray Circular Dichroism and Local Magnetic Fields, Phys. Rev. Lett. 70, 694 (1993).

[57] T. Jo, The $3 d-4 f$ Exchange Interaction, X-Ray Second-Order Optical Processes, and the Magnetic Circular Dichroism (MCD) Spin Sum Rule in Rare Earths, J. Electron Spectrosc. Relat. Phenom. 86, 73 (1997).

[58] Y. Nakajima, P. S. Syers, X. F. Wang, R. Wang, and J. Paglione, One-Dimensional Edge State Transport in a Topological Kondo Insulator, arXiv:1312.6132.

[59] B. Gorshunov, N. Sluchanko, A. Volkov, M. Dressel, G. Knebel, A. Loidl, and S. Kunii, Low-Energy Electrodynamics of $\mathrm{SmB}_{6}$, Phys. Rev. B 59, 1808 (1999). 